\section{Guideline für Guidelines}

\author{
H. H. Brunner
}

Möglicherweise war es als leise Provokation gedacht, mich an diesem Symposium zum Thema «Guideline für Guidelines» sprechen zu lassen. Die von der FMH publizierte "Guideline für Guidelines» [1] hat ja recht lebhafte Diskussionen ausgelöst. Allerdings werde ich jetzt nicht über diese Guideline als solche sprechen, sondern vielmehr deren Hintergründe und Zielsetzungen beleuchten.

\section{Grundprinzipien}

Henry Mintzberg, einer der Gurus der Managementwissenschaften, hat vor kurzem, an der «3rd Conference for Health" in Toronto, vier Positionen formuliert, die mir im Kontext der Guidelines als zentral erscheinen und die ich deshalb an den Beginn meiner Ausführungen stellen möchte.

1. Medicine is not a science; medicine is not applied science, medicine is the application of science - among other things.

Hier wird gewarnt vor der Illusion, dass man die Medizin aus irgendwelchen naturwissenschaftlichen Erkenntnissen gleichsam deduktiv bis ans Krankenbett ableiten könne.

2. Work is professional precisely because it requires nuanced judgement, which means that while its costs may easily be measured its benefits often cannot. Das ist die Situation, in der wir im Bezug auf die Guidelines stehen. Wenn es darum geht, den Nutzen einer Guideline oder einer Behandlung zu beurteilen, sollten nicht nur die direkten Kosten betrachtet werden; ebenso wichtig ist die Frage nach dem Benefit, die aber leider häufig nicht schlüssig beantwortet werden kann.

3. The management of the clinical operations has to be done by the managed, not by the managers.

Das ist ein ausserordentlich hintergründiger Satz, weil häufig nicht ganz klar ist, wer eigentlich die "managers" sind und wer die "managed". Ich interpretiere ihn so, dass eine Berufsorganisation (z.B. die FMH) ihren Mitgliedern nicht vorschreiben kann, wie

Korrespondenz:

Dr. med. Hans Heinrich Brunner

Präsident FMH

Elfenstrasse 18

CH-3000 Bern 16 sie agieren sollen. Für uns war dies ein absoluter Grundsatz: Wir wollen nicht die Manager sein, sondern wir wollen Hilfestellungen geben für diejenigen, die am Krankenbett den Prozess managen müssen.

4. Hospitals don't need "bosses" or "professional style of managing"; they need a craft style, more akin to care than to cure

Darin liegt die Grundphilosophie, mit der wir versucht haben, an die Guidelines und auch an diese Guideline für Guidelines heranzutreten.

"Ownership of problems" ist nicht gleichbedeutend mit "ownership of solutions". Auch diese Feststellung hat uns bei unserer Arbeit geleitet. Auf die Guideline bezogen bedeutet sie, dass Gruppen und Leute, die ein Problem sehen, nicht notwendigerweise über die Instrumente verfügen, das Problem zu lösen. Es braucht die Zusammenarbeit mit jenen, die z.B. methodisch den Prozess der Guidelineentwicklung beherrschen.

Wenn wir von Guideline sprechen, ist immer die erste Frage, wer der Adressat ist. Sind es die Patienten, die Politiker, die Kostenträger, die Spitalverwaltungen oder die Ärztinnen und Ärzte? Die FMH hat den Grundsatzentscheid getroffen, dass sie sich mit ihrer Guideline für Guidelines bzw. ihren Guidelines, für die sie sich verantwortlich fühlt, an die Ärztinnen und Ärzte wendet. Das ist auch eine bewusste Selbstbeschränkung, die wir hier vollzogen haben.

\section{Implementierung und Akzeptanz}

Bei der Erstellung von Guidelines ist die Frage der richtigen Evidenz ein grosses Problem. Aber von mindestens ebenso grosser Tragweite ist das Problem der Implementierung: Gelingt es, gute Guidelines so zu implementieren, dass sie auch angewandt werden? Ist es nur die Evidenz, die massgeblich ist, oder gibt es noch andere Faktoren, z.B. ein Konsensusprozess, die einen Einfluss haben? Ein Konsensusprozess hat nicht nur die Funktion, Wissen aus den Leuten herauszuholen, das man sonst nicht bekommt, sondern er kann auch mithelfen, dass der praktizierende Arzt, die praktizierende Ärztin eine Guideline akzeptieren. Man weiss z.B., dass das Prinzip des "peers" oder des "opinion leaders" absolut zentral ist: Wenn ich den Kollegen X für einen guten Arzt halte, bin ich eher geneigt, von ihm zu lernen, als wenn ich ihn als unfähig ansehe - auch wenn die Evidenz die gleiche ist.

Das führt zum nächsten Punkt, der absolut zentralen Frage der Akzeptanz dieser Guidelines in ärztlichen Kreisen. Es sind einige Faktoren, die ich etwas näher erläutern möchte. Der Akzeptanz hinderlich ist der Vorwurf, es handle sich bei den Guidelines um eine Kochbuchmedizin, und es drohe damit der Verlust der professionellen Autonomie. Diesen Vorwurf muss man sehr ernst nehmen.

Ein weiterer wichtiger Faktor, der sich mit dem ersten etwas überlappt, besteht darin, dass der Arzt häufig das, was in der Guideline steht, als theoretisch empfindet, verglichen mit dem, was er am Kranken- 
bett erlebt. Das führt dann oft zum Schluss, dass es in der täglichen Arbeit nicht hilft und links liegengelassen wird.

Es sind auch Ängste mit den Guidelines verbunden, sehr wichtige und tiefgreifende Ängste, z.B. die Angst vor Kontrolle und Überwachung. In diesem Prozess, den wir jetzt begonnen haben, ist dies sehr eindrücklich zum Ausdruck gekommen. Diese Angst muss ernst genommen werden, um so mehr, als sie mit einer zweiten Angst verbunden ist, derjenigen vor juristischen Konsequenzen. Auch diese Angst hat durchaus ihre realen Begründungen, wie die Beispiele aus den Vereinigten Staaten und Grossbritannien zeigen.

Es gibt ein weiteres Element, das mir sehr wichtig erscheint: Die Basis für das, was in die Guidelines einfliesst - d.h. die Evidenz - ist eine statistische, epidemiologisch fundierte Medizin. Und die steht in fast konträrem Gegensatz zu einer Medizin, wie wir sie am Krankenbett erleben, die einen extrem narrativen Charakter hat; wir Ärztinnen und Ärzte leben aus dem Fall, den wir erlebt haben.

\section{Alternativen}

Man könnte sich jetzt sagen, wenn die Widerstände so gross sind, vergessen wir doch die Guidelines, hören wir auf, suchen wir andere Wege. Es gibt diesbezüglich durchaus Ideen und Vorschläge, dass z.B. jeder Arzt das nötige "critical appraisal" des gesamten Wissens selbst betreibt. Einer solchen Anforderung kann wahrscheinlich kaum jemand genügen, und sie ist nur denkbar in einem sektoriell engen Gebiet, nicht jedoch in der Hausarztmedizin.

Eine weitere Möglichkeit sind die "critical reviews", wo Ärztinnen und Ärzte für einen bestimmten Bereich die vorhandene Evidenz zu gewichten versuchen. Allerdings hält sich die Verfügbarkeit des so geschaffenen Wissens in Grenzen, vor allem dann, wenn ich nachts um 2 Uhr am Krankenbett einen Entscheid fällen muss. Und die Ärztinnen und Ärzte stehen immer oder sehr häufig unter einem sehr grossen Zeitdruck, unter dem sie entscheiden müssen. Es ist denkbar, dass diese Methode durchaus eine Bedeutung erlangt in dem Ausmasse, wie uns elektronisch abrufbares Wissen zur Verfügung steht. Dem gegenüber steht jedoch eine geradezu explosive Vermehrung des Wissens, welche die mit diesen Techniken erzielten Gewinne gleichsam wieder auffrisst. Ein weiteres grundsätzliches Problem besteht darin, dass diese Reviews je nach Autor zu verschiedenen Resultaten kommen. Und dann stehe ich auch wieder, um einen der Klassiker zu zitieren, «als Tor [vor der Situation] und bin so klug als wie zuvor».

\section{Politische Probleme}

Als Berufsorganisation müssen wir uns aber nicht nur mit internen Problemen bzw. Problemen, die von unseren Kolleginnen und Kollegen kommen, auseinan- dersetzen, sondern wir sollten auch andere Entwicklungen im Auge behalten. Diese mehr politischen Probleme möchte ich unter folgende Headline stellen: Wenn wir uns in dieser Sache nicht engagieren, werden es andere tun bzw. tun sie es im Grunde schon in einem gefährlichen Ausmasse. Die Interferenz des ärztlichen Handelns mit anderen Interessen ist offensichtlich. Dies führt dazu, dass Pharmaindustrie, Versicherer oder Managed-Care-Organisationen versucht sind, mit selbst entwickelten Guidelines die Art und Weise von Therapien zu beeinflussen. Eine ausserordentlich kritische Dimension gewinnt ein solches Zusammenspiel dort, wo es um alle Fragen der Rationierung geht.

Wir leben davon, dass ungefähr alle zwei Jahre ein neues Schlagwort im Management des Gesundheitswesens auftritt. Im Moment ist dies die «clinical governance" - eine raffinierte Form, wie sich Politiker aus der Verantwortung stehlen und der Klinik sagen, ihr seid jetzt für das gesamte Management verantwortlich. In diesem Kontext ist es ausserordentlich wichtig, über irgendwelche Managementinstrumente $\mathrm{zu}$ verfügen. Und hier bieten sich natürlich Guidelines geradezu an. Wenn dann die Menschen, die wirklich am Krankenbett arbeiten, nicht begreifen, wie diese Prozesse ablaufen, kann es sein, dass sie plötzlich - auf einem strategisch sehr niedrigen Niveau - mit sehr konkreten Vorgaben konfrontiert werden in einer Art und Weise, wie das viel gefährlicher ist, als wenn solche Guidelines von einem höheren strategischen Niveau kommen.

Das andere Schlagwort ist das "disease management", wo sich bereits verschiedene Versicherer, aber auch pharmazeutische Industrien in den letzten Jahren engagiert haben, praktisch an der Ärzteschaft vorbei. Das sind die Realitäten, und diese Realitäten müssen wir wahrnehmen, wir müssen mit ihnen umgehen und uns positionieren.

Eine letzte - und wahrscheinlich die gefährlichste - Entwicklung ist die, dass die Guidelines als Raster für das "quality profiling" dienen sollen. Dass versucht wird, nach irgendwelchen kruden Kriterien zu entscheiden, welches die guten Ärzte sind und welches die schlechten, die aus dem System gekippt werden. Die aktuelle KVG-Revision will dieses "quality profiling” im Gesetz verankern. Und jetzt suchen die Leute nach Instrumenten, mit denen sie dieses "quality profiling" bei den Ärztinnen und Ärzten durchführen können. Das hat alles hoch realen Charakter, das ist nicht etwas, was irgendeinmal in den nächsten Jahren passieren wird.

\section{Offene Fragen}

Ich möchte jetzt noch einige Fragen ansprechen, die offengeblieben sind, auch in dieser "Guideline für Guidelines", die in der Schweizerischen Ärztezeitung publiziert worden ist. Es war dies gleichsam eine erste Version, welche sicher noch weiter verbessert werden muss. Das war uns klar und wurde so auch in der Schweizerischen Ärztezeitung dargelegt. 
- Soll man - was bisher nicht geschehen ist - den gesamten Bereich der Medizinökonomie, also z.B. Cost-effectiveness-Fragen, in eine solche Guideline aufnehmen?

- Sollen die Patienten bzw. ihre Organisationen in diesen Prozess des Aufbaus einer Guideline einbezogen werden?

- Wie wird das Problem der Verwaltung von Guidelines, das "updating", gelöst?

- Wer trägt die immensen Kosten, die vor der Türe stehen, wenn man sich effektiv engagieren will?

- In welchem Ausmass können wir ausländische Guidelines in der Schweiz übernehmen?

- Gibt es bei schweizerischen Guidelines, auf schweizerischer Ebene, allenfalls regionale Module, die z.B. die Applikation in der Westschweiz und in der deutschen Schweiz modifizieren?

- Geht der Weg von der klassischen Guideline immer mehr zum «decision making pathway»?
Einer der auslösenden Faktoren für das Engagement der FMH war die mangelnde Qualität vieler der publizierten Guidelines und der Wunsch, hier Ordnung in das System zu bringen. Bei diesem Projekt sind wir auf sehr intensive Zusammenarbeit angewiesen, z.B. mit der Schweizerischen Akademie der Medizinischen Wissenschaften, mit welcher z.Z. entsprechende Gespräche geführt werden. Mit anderen Worten spreche ich hier auch eine Einladung aus, mitzuarbeiten an einem Projekt, das nicht einer allein bewerkstelligen kann.

\section{Literatur}

1 Eicher E. Guideline für Guidelines. Schweiz Ärztezeitung 1999;80(10):581-4. 